•研究简报・

\title{
贵州花溪大学城破碎化林地鸟类多样性与嵌套分 布格局
}

郑进凤 ${ }^{1}$, 唐蓉 ${ }^{1}$, 贺霜 ${ }^{1}$, 陈月红 ${ }^{1}$, 伍素 ${ }^{1}$, 张凯 ${ }^{1}$, 徐雨 ${ }^{(1} 1^{*}$, 邹晓 ${ }^{2}$

1. 贵州师范大学生命科学学院, 贵阳 550025 ; 2. 贵州大学生命科学学院, 贵阳 550025

摘要: 为揭示城镇化进程中生境破碎化对鸟类多样性及分布格局的影响, 本研究于2017-2019年每年的4-8月使用样线法对 贵州花溪大学城26块破碎化林地(面积介于 $0.3-290.4 \mathrm{ha}$ )中的鸟类群落进行了 10次调查。共记录到鸟类78种, 隶属于11目37 科。其中, 东洋界物种数占 $56.4 \%$, 古北界物种数占 $32.1 \%$, 广布种占 $11.5 \%$; 有中国特有种 1 种。剔除高空飞行、非森林鸟类 及偶然出现物种后，不同斑块中的鸟类物种数介于12-55之间，平均每个斑块有 $23.2 \pm 10.5$ 种。线性回归分析显示，鸟类物种 丰富度与林地斑块的面积有显著相关性，斑块面积越小，鸟类物种丰富度越低; 斑块隔离度对物种丰富度没有显著影响。基 于物种多度分布矩阵的WNODF (weighted nestedness metric based on overlap and decreasing fill)嵌套分析显示, 不同斑块中鸟 类群落呈现出反嵌套结构。小斑块中鸟类物种丰富度较低可能与植物丰富度较低、食物资源稀缺和繁育条件不足有关, 但短 距离的隔离对鸟类迁入或扩散影响有限。环境过滤效应、种间竞争或优先效应可能导致不同斑块间存在较大的物种组成差异, 从而导致反嵌套格局。因此，本研究建议在城市规划建设中应注重维持栖息地的完整性，对不同面积大小的破碎化斑块都应 加以保护。

关键词: 斑块; 面积; 隔离度; 嵌套格局; 物种丰富度; 生物多样性; 城市化

郑进风, 唐蓉, 贺霜, 陈月红, 伍素, 张凯, 徐雨, 邹晓 (2021) 贵州花溪大学城破碎化林地鸟类多样性与嵌套分布格局. 生物多样性, 29, 661-667. doi: 10.17520/biods.2020336.

Zheng JF, Tang R, He S, Chen YH, Wu S, Zhang K, Xu Y, Zou X (2021) Bird diversity and nestedness on fragmented woodlots in Huaxi University Town, Guizhou Province. Biodiversity Science, 29, 661-667. doi: 10.17520/biods.2020336.

\section{Bird diversity and nestedness on fragmented woodlots in Huaxi University Town, Guizhou Province}

\author{
Jinfeng Zheng ${ }^{1}$, Rong Tang ${ }^{1}$, Shuang $\mathrm{He}^{1}$, Yuehong $\mathrm{Chen}^{1}$, Su Wu${ }^{1}$, Kai Zhang ${ }^{1}$, Yu Xu ${ }^{\mathbb{D}_{1 *}}$, Xiao Zou ${ }^{2}$
}

1 School of Life Sciences, Guizhou Normal University, Guiyang 550025

2 School of Life Sciences, Guizhou University, Guiyang 550025

\section{ABSTRACT}

Aim: To examine the impact of habitat fragmentation stemming from urbanization on bird diversity and distribution patterns.

Methods: We surveyed bird assemblages on 26 fragmented woodlots with areas of 0.3-290.4 ha in Huaxi University Town, Guizhou Province from April to August between 2017 and 2019. We surveyed birds by establishing line transects in each woodlot, with transect length roughly proportional to the woodlot area. We surveyed each line transect 10 times over the course of the study period, and recorded birds detected within $50 \mathrm{~m}$ of either side of the line transects.

Results: In total, we recorded 78 bird species belonging to 11 orders and 37 families. Species of Oriental origin, Palaearctic origin, and widespread species accounted for $56.4 \%, 32.1 \%$, and $11.5 \%$ of observations, respectively. We observed one species endemic to China. After excluding birds detected as fly-overs (e.g., swallows and swifts), non-forest dwelling birds (e.g., waterbirds), and birds that were only recorded once in the survey, we found that the number of bird species in different woodlots ranged from 12 to 55 species, with an average of $23.2 \pm 10.5$ species per

收稿日期: 2020-08-20; 接受日期: 2020-12-27

基金项目: 国家自然科学基金委员会-贵州省人民政府喀斯特科学研究中心项目(U1812401)

* 通讯作者 Author for correspondence. E-mail: xuyu608@gznu.edu.cn 
woodlot. Linear regression analysis showed that bird species richness was significantly positively correlated with woodlot area, with larger woodlots having higher bird richness. A metric of isolation, measured as the distance to the nearest neighboring woodlot, had no significant effect on bird species richness. The analysis of nestedness, based on the metric WNODF (weighted nestedness metric based on overlap and decreasing fill), conducted on the species-by-site abundance matrix, revealed that the observed WNODF for sites of birds was significantly lower than expected from the null model, indicating that bird assemblages were anti-nested among the woodlots.

Conclusion: Our results suggest that plant richness, food resources and breeding conditions in small woodlots may be diminished compared to larger woodlots, making it difficult for some bird species to meet their survival needs. However, the relatively short distance between woodlots in our study sites combined with the stronger dispersal ability of birds may mask the biological importance of isolation. The anti-nested structure of bird communities may be the result of larger differences in species composition between woodlots being driven by environmental filtering, interspecific competition, or priority effects, but further research is needed to determine the likely causal mechanism of anti-nestedness. Nevertheless, given that rapid urban expansion has caused substantial habitat fragmentation in recent decades in our study area, we recommend that habitat integrity should be prioritized and maintained in urban planning when possible, and that fragmented woodlots of different sizes be protected.

Key words: patch; area; isolation; nestedness; species richness; biodiversity; urbanization

生境破碎化是指一片连续的生境由于人类活 动或自然过程被缩小、分割为多个面积不等、彼此 隔离的小斑块的现象(Fahrig, 2003)。研究生境破碎 化对生物多样性的影响, 是生态学和保护生物学的 重要课题之一(张明海和马建章, 2014; 吴倩倩等, 2017; Miller-Rushing et al, 2019)。

陆桥岛屿、森林斑块和城市绿地是常被研究的 破碎化生境。早期研究主要依据岛屿生物地理学理 论(Wilson \& MacArthur, 1967)分析生境破碎化后不 同面积和隔离度的斑块内物种丰富度的差异。通常 认为, 面积是影响物种丰富度的最重要因素 (Watling \& Donnelly, 2006; 吴倩倩等, 2017)。在面 积较小的斑块中, 由于空间范围较小、生境多样性 较低、食物资源量较少, 一些物种难以生存, 因此 物种丰富度较低(面积效应)。生境破碎化后的斑块 往往也表现出不同程度的地理隔离, 影响物种迁 移、扩散和基因流交换, 导致物种丰富度随斑块隔 离度的增加而减少(隔离效应)。目前, 大量案例分析 证实了面积效应(Watling \& Donnelly, 2006; 吴倩倩 等, 2017; Miller-Rushing et al, 2019)。但是, 隔离效 应是否存在尚有一定争议。比如, 鸟类具有极强的 扩散能力, 斑块隔离度可能对其物种丰富度没有影 响或影响很小(Watling \& Donnelly, 2006; 吴奕如等, 2016)。

除了物种丰富度外, 学者也从群落组成与变化 角度分析了生境破碎化对生物多样性的影响。其中, 嵌套分析是研究群落物种组成和分布格局最常用 的手段(Wang et al, 2010)。嵌套格局是由Darlington
(1957)提出的物种分布模式, 它描述低丰富度斑块 中的物种是高丰富度斑块中物种集合的子集。通常 认为, 嵌套格局的产生与被动取样(Andrén, 1994)、 物种选择性灭绝(Patterson \& Atmar, 1986)、选择性 迁入(Cook \& Quinn, 1995)及生境嵌套(Blake, 1991) 有关。目前, 已有不少学者报道了破碎化生境中的 动物群落呈现嵌套格局, 并从栖息地特征、物种生 活史特征等角度验证了上述的假说(张竞成等, 2008; Wang et al, 2013; Chen et al, 2019; Li et al, 2019)。但 是, 一些研究也指出, 受竞争作用、环境过滤 (environmental filtering)、优先效应(priority effect)等 因素的影响, 破碎化生境中的动物群落可能不会嵌 套, 甚至呈现出反嵌套结构(Gutiérrez-Cánovas et al, 2013; Matthews et al, 2015; Chen et al, 2018)。

近年来, 由城镇发展导致的生境破碎化现象日 益严重(Liu et al, 2019)。城市破碎化生境主要是指 被建筑物和人工设施分割的绿地或公园, 它们与陆 桥岛屿相比情况可能更加复杂, 遭受人类干扰的程 度也较大(Fernández-Juricic, 2002; González-Oreja et al, 2012; Wang et al, 2013)。为了进一步丰富有关城 市生境破碎化的研究内容, 合理指导城市规划、建 设和实施物种保护, 本文对贵州省花溪大学城破碎 化自然林地中的鸟类多样性和嵌套分布格局进行 了研究。

\section{1 研究方法}

\section{1 研究区概况}

研究区位于贵州省贵安新区东南部, 紧邻贵阳 
市花溪区 $\left(26^{\circ} 22^{\prime}-26^{\circ} 24^{\prime} \mathrm{N}, 106^{\circ} 36^{\prime}-106^{\circ} 40^{\prime} \mathrm{E}\right.$; 图1), 面积约 $10 \mathrm{~km}^{2}$ 。该区域为喀斯特地貌，海拔约 $1,200 \mathrm{~m}$, 属于亚热带湿润温和型气候, 年平均气温 约为 $15^{\circ} \mathrm{C}$, 年平均降水量约为 $1,130 \mathrm{~mm}$ 。大学城修 建以前，这片区域为农田环绕的自然林地。自2009 年大学城动工修建至后期投入使用, 自然林地进一 步被建筑物、道路所分割。残留的林地斑块中的植 被相对比较简单, 主要由暖性松林、常绿落叶阔叶 林、落叶阔叶灌从和禾草灌草丛组成。常见的乔木 有马尾松(Pinus massoniana)、锐齿槲柇(Quercus aliena var. acutiserrata)、化香 树 (Platycarya strobilacea)等; 灌木有火棘(Pyracantha fortuneana)、 马桑(Coriaria nepalensis)、小果菩薇(Rosa cymosa) 等; 形成群系的草本主要有芒 (Miscanthus sinensis)、狗尾草(Setaria viridis)等。本研究选择破 碎化景观中的 26 个斑块为研究样地, 面积介于 0.3-290.4 ha (图1, 附录1)。

\section{2 野外调查}

从 Google地球下载研究区的卫星影像, 使用 ArcGIS 10.2勾勒出每个斑块的边界, 计算面积。同 时, 计算每个斑块边缘到最近斑块边缘的距离, 以 此衡量其隔离度(吴奕如等, 2016; Xu et al, 2017)。在 每个斑块中设置长度与斑块面积成正比的样线(样 线数量和长度见附录 1 ; 样线长度与斑块面积的相 关系数 $r=0.99$ ), 样线尽可能穿越斑块的中心点, 以减少取样不均衡和取样偏差等问题(斯幸峰,
2014)。在2017-2019年每年的4-8月, 选择无雨、无 雾的晴好天气进行鸟类调查。平均每1.5-2个月开展 1 轮调查, 共计开展 10轮(2017-2018年每年3轮, 2019年4轮)。一般 由 2 名固定人员协作完成一轮调查 后，轮换 2 名人员继续开展下一轮，前后共计 14 人 参与调查。调查时间为鸟类活动相对频繁的 7:00-10:00和16:00-18:00。在调查时, 调查者在样线 上的行进速度为 $1-1.5 \mathrm{~km} / \mathrm{h}$, 使用 $10 \times 42$ 双筒望远 镜观测样线两侧 $50 \mathrm{~m}$ 范围内的鸟类, 记录物种、数 量、行为等信息。野外鸟类识别参考《中国鸟类野 外手册》(约翰・马敬能等, 2000)等工具书。

\section{3 数据分析}

依据《中国鸟类分类与分布名录(第三版)》(郑 光美, 2017)的分类系统, 统计研究区的总体鸟类组 成。按照《中国动物地理》统计鸟类的区系组成(张 荣祖, 2011)。然后，剔除燕子、雨燕、水鸟等高空 飞行或非森林鸟类以及调查中仅记录到1次的偶然 出现物种 (Chen et al, 2018; Wagner \& Reynolds, 2019), 分析 26 个林地斑块中鸟类物种丰富度特征 和嵌套分布格局。在R.4.0.2中, 利用 $\operatorname{lm}$ 函数构建物 种丰富度与斑块面积、距最近斑块距离的线性回归 模型，使用 visreg程序包绘制模型的偏残差图 (Breheny \& Burchett, 2020)。

将10次调查的数据累积构建斑块与鸟类物种 多度矩阵, 使用WNODF (weighted nestedness metric based on overlap and decreasing fill)方法分析

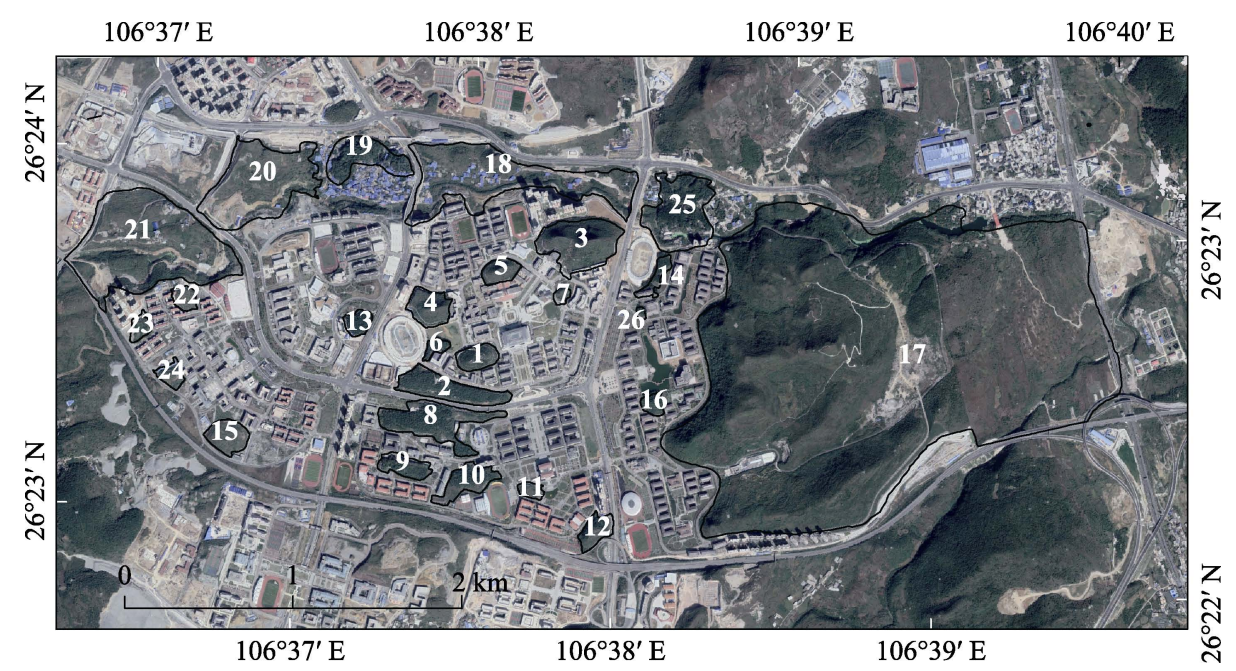

图1 贵州花溪大学城的位置及Google卫星影像下 26 个破碎化林地斑块的分布

Fig. 1 Google map imagery showing location of the study site and distribution of 26 fragmented woodlots in Huaxi University Town, Guizhou Province 
鸟类群落的嵌套格局 (Almeida-Neto \& Ulrich, 2011)。与其他方法相比, WNODF法不仅可以对整 个矩阵进行分析, 还能分别计算物种(WNODFr) 和 斑块(WNODFc)的嵌套值。此外, 该方法对矩阵大 小和填充度不敏感, 且能有效避免统计错误。在 NODF2.0软件中执行数据分析 (Almeida-Neto \& Ulrich, 2011); 为避免统计错误, 同时设置行列固定 (rc)、总数固定 $(\mathrm{aa})$ 及总物种丰富度固定 $(\mathrm{ss})$ 等 3 种重 采样零模型, 依据软件随机产生1,000个矩阵, 对输 入矩阵的嵌套程度进行显著性检验。

在上述统计分析中, $P<0.05$ 时视为具有统计显 著意义。

\section{2 结果}

\section{1 物种组成}

在 10 次调查中, 共记录到鸟类78种, 隶属于 11 目 37 科 (附录 2)。从区系来看, 东洋界物种数占 $56.4 \%$, 古北界占 $32.1 \%$, 广布种占 $11.5 \%$ 。有中国特 有种1种, 即乌冻(Turdus mandarinus)。

\section{2 物种丰富度}

在剔除燕子、雨燕、水鸟等高空飞行或非森林 鸟类以及调查中仅记录到 1 次的偶然出现物种后, 统计发现不同斑块中鸟类物种数介于 $12-55$, 平均 每个斑块有 $23.2 \pm 10.5$ (mean $\pm \mathrm{SD}$ ) 种。线性回归分
析表明(图2), 鸟类物种丰富度与斑块面积呈显著正 相关 $(\beta=0.20, P<0.001)$, 与距最近斑块距离没有 显著相关性 $(\beta=-0.07, P=0.150)$ 。

\section{3 嵌套格局}

采用 $r c$ 和 $a \mathrm{a}$ 零模型的WNODF嵌套分析显示 (表1), 26 个林地斑块中鸟类物种多度矩阵的嵌套程 度显著低于随机产生的 1,000 个分布矩阵( $\mathrm{rc}$ 零模型: $Z=-4.36, P<0.001$; aa零模型: $Z=-3.71, P<0.001$ ), 表明存在显著的反嵌套结构。其中, 物种( $\mathrm{rc}$ 零模型: $Z=-4.83, P<0.001$; aa零模型: $Z=-3.65, P<0.001)$ 对这一分布格局的贡献高于林地斑块(rc零模型: $Z$ $=-0.61, P=0.272$; aa零模型: $Z=-1.62, P=0.053)$ 。 ss 零模型分析的结果表明(表1), 26 个林地斑块中鸟 类群落存在近显著性的反嵌套结构 $(Z=-1.36, P=$ $0.088)$, 物种 $(Z=-1.41, P=0.080)$ 对这一分布格局 的贡献也高于林地斑块 $(Z=-0.88, P=0.189)$ 。

\section{3 讨论}

本研究发现，在贵州花溪大学城 $10 \mathrm{~km}^{2}$ 的区域, 春夏季记录到鸟类78种, 占贵州省已知鸟类种数 (贵州省环境科学研究设计院，2010)的15.4\%。数据 分析表明鸟类物种丰富度与林地斑块的面积呈显 著正相关，符合岛屿生物地理学理论提出的“面积 效应” (Wilson \& MacArthur, 1967)。由于城镇建设,

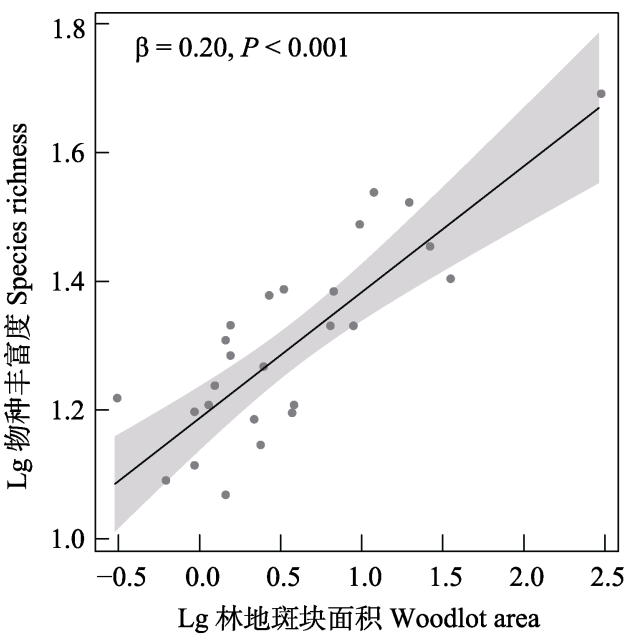

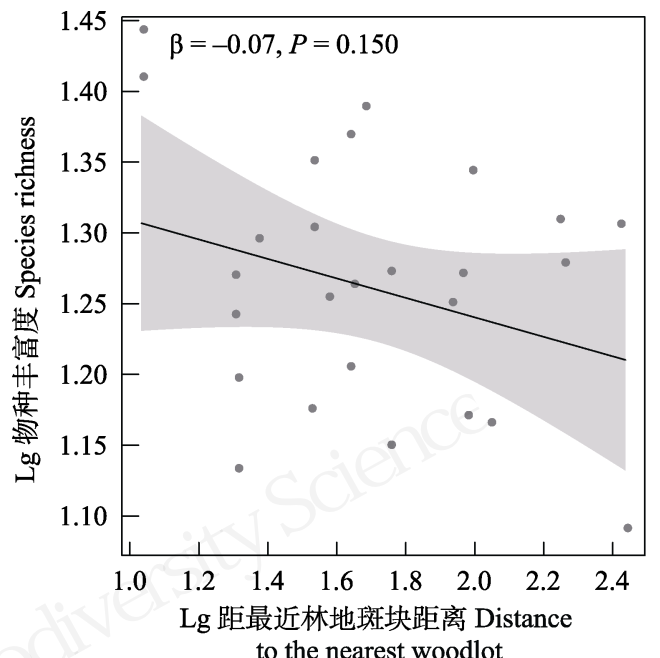

图2 贵州花溪大学城26个破碎化林地中鸟类物种丰富度(10为底对数转换)与林地斑块面积(10为底对数转换; 单位: ha)和距 最近斑块距离(10为底对数转换; 单位: $m$ )的线性回归偏残差图。黑线表示拟合值, 灰色区域表示 $95 \%$ 置信区间。

Fig. 2 Partial residuals showing the effect of woodlot area (Lg transformed, unit: ha) and distance to the nearest woodlot (Lg transformed, unit: m) on species richness (Lg transformed) of birds recorded on 26 fragmented woodlots in Huaxi University Town, Guizhou Province, according to the linear regression analyses. The black lines are model fits, and the grey bands represent $95 \%$ confidence intervals. 
表1 贵州花溪大学城26个破碎化林地中鸟类物种多度矩阵 的嵌套格局分析结果

Table 1 Results of nestedness analyses for species-by-sites abundance matrix of birds recorded on 26 fragmented woodlots in Huaxi University Town, Guizhou Province

\begin{tabular}{|c|c|c|c|c|c|}
\hline $\begin{array}{l}\text { 嵌套度量化 } \\
\text { Nestedness metric }\end{array}$ & $\begin{array}{l}\text { 观测值 } \\
\text { Observed }\end{array}$ & $\begin{array}{l}\text { 期望值 } \\
\text { Expected }\end{array}$ & $\begin{array}{l}\text { 标准差 } \\
\mathrm{SD}\end{array}$ & $Z$ & $P$ \\
\hline \multicolumn{6}{|c|}{ 零模型 Null model: rc } \\
\hline WNODF & 45.92 & 51.36 & 1.25 & -4.36 & $<0.001$ \\
\hline WNODFc & 46.98 & 48.00 & 1.68 & -0.61 & 0.272 \\
\hline WNODFr & 45.71 & 52.05 & 1.31 & -4.83 & $<0.001$ \\
\hline \multicolumn{6}{|c|}{ 零模型 Null model: aa } \\
\hline WNODF & 45.92 & 51.75 & 1.57 & -3.71 & $<0.001$ \\
\hline WNODFc & 46.98 & 49.61 & 1.63 & -1.62 & 0.053 \\
\hline WNODFr & 45.71 & 52.2 & 1.78 & -3.65 & $<0.001$ \\
\hline \multicolumn{6}{|c|}{ 零模型 Null model: ss } \\
\hline WNODF & 45.92 & 53.37 & 5.50 & -1.36 & 0.088 \\
\hline WNODFC & 46.98 & 50.31 & 3.78 & -0.88 & 0.189 \\
\hline WNODFr & 45.71 & 54.01 & 5.90 & -1.41 & 0.080 \\
\hline
\end{tabular}

自然林地被道路与房屋等人工设施进一步分割、缩 小。小斑块中的生境丰富度和资源量可能较低, 难 以满足一些物种的生存需求, 因此鸟类物种丰富度 较低(王本耀等, 2012; Wang et al, 2013; De la Hera, 2019)。但是, 本研究发现鸟类物种丰富度与最近斑 块距离的相关性不显著, 表明不存在隔离效应。本 研究区内不同斑块之间隔离度较低, 一些斑块仅仅 只被一条公路所隔离。再者, 破碎的自然林地斑块 间镶嵌有人工绿地或栽培有行道树, 一定程度上也 可能减弱隔离程度。由于鸟类具有极强的飞行能力, 短距离的隔离对其迁入或扩散影响有限，因此没有 产生隔离效应(Ewers \& Didham, 2006; Watling \& Donnelly, 2006; 吴奕如等, 2016)。

进一步分析揭示, 不同林地斑块间鸟类群落呈 现出反嵌套结构, 暗示斑块间具有较高的物种替 换。该结果与大多数城市的破碎化生境的研究结果 不同(王本耀等, 2012; González-Oreja et al, 2012; Wang et al, 2013; De la Hera, 2019)。一般认为, 嵌套 格局的产生与选择性迁入(Cook \& Quinn, 1995)、选 择性灭绝(Patterson \& Atmar, 1986)和生境嵌套 (Blake, 1991)有关。由于本研究区内不同林地斑块 之间隔离度较低, 对鸟类难以形成阻隔, 因此出现 选择性迁入的可能性不大。选择性灭绝假说认为在 生境破碎化过程中，一些对面积敏感的物种会率先
在小斑块中消失，从而导致嵌套格局出现(Patterson \& Atmar, 1986)。虽然本研究揭示鸟类物种丰富度随 林地斑块面积减小而降低，但强烈的种间竞争作用 (比如，繁殖期鸟类竞争巢址、防护领域等)可能造成 单纯通过面积无法有效解释的斑块间物种组成差 异，从而削弱嵌套格局或导致反嵌套格局 (Matthews et al, 2015; Chen et al, 2018)。

由于缺乏调查，尚不清楚不同斑块间是否存在 生境嵌套。虽然本研究区植被组成相对简单, 但喀 斯特特殊的地质地貌可能造成局部小生境和植物 种类差异(调查已发现不同斑块中木本植物的组成 差异较大)。在生境破碎化过程中, 物种受小生境和 植物种类差异的篮选，出现在各自适应的特定生境 中，即环境过滤作用(Gutiérrez-Cánovas et al, 2013), 从而导致反嵌套格局出现。再者，优先效应也可能 导致反嵌套格局产生。当一些物种优先占据某一特 定生境后, 可能排斥后续到达的物种, 进而导致不 同斑块间具有较高的物种替换(Fukami, 2015; Chen et al, 2018)。究竟是什么机制引发反嵌套格局的形 成还有待进一步研究。

综上, 本研究建议在城市规划建设中应注重维 持鸟类栖息地的完整性，对不同面积的破碎化林地 斑块都应加以保护。

致谢: 感谢龙盛尧、再小东、田佼、邰合、朱芸、 任云芳、雷小琴等同学在野外鸟类调查工作中所付 出的努力。

\section{ORCID}

徐雨 (D) https://orcid.org/0000-0002-9437-3267

\section{参考文献}

Almeida-Neto M, Ulrich W (2011) A straightforward computational approach for measuring nestedness using quantitative matrices. Environmental Modelling \& Software, 26, 173-178.

Andrén H (1994) Can one use nested subset pattern to reject the random sample hypothesis? Examples from boreal bird communities. Oikos, 70, 489-491.

Blake JG (1991) Nested subsets and the distribution of birds on isolated woodlots. Conservation Biology, 5, 58-66.

Breheny P, Burchett W (2020) Package 'visreg' Version 2.7.0. https://cran.r-project.org/web/packages/visreg/. (accessed on 2020-10-25) 
Chen CW, Holyoak M, Si XF, Wang YP, Ding P (2018) Do seasonal species assemblages differ in their biogeography? Evidence from the spatial structure of bird communities on land-bridge islands. Journal of Biogeography, 45, 473-483.

Chen CW, Xu AC, Ding P, Wang YP (2019) The small-island effect and nestedness in assemblages of medium- and large-bodied mammals on Chinese reservoir land-bridge islands. Basic and Applied Ecology, 38, 47-57.

Cook RR, Quinn JF (1995) The influence of colonization in nested species subsets. Oecologia, 102, 413-424.

Darlington PJ (1957) Zoography: The Geographical Distribution of Animals. John Wiley \& Sons, New York.

De la Hera I (2019) Seasonality affects avian species distribution but not diversity and nestedness patterns in the urban parks of Vitoria-Gasteiz (Spain). Animal Biodiversity and Conservation, 42, 279-291.

Ewers RM, Didham RK (2006) Confounding factors in the detection of species responses to habitat fragmentation. Biological Reviews, 81, 117-142.

Fahrig L (2003) Effects of habitat fragmentation on biodiversity. Annual Review of Ecology, Evolution, and Systematics, 34, 487-515.

Fernández-Juricic E (2002) Can human disturbance promote nestedness? A case study with breeding birds in urban habitat fragments. Oecologia, 131, 269-278.

Fukami T (2015) Historical contingency in community assembly: Integrating niches, species pools, and priority effects. Annual Review of Ecology, Evolution, and Systematics, 46, 1-23.

González-Oreja JA, De La Fuente-Díaz-Ordaz AA, Hernández-Santín L, Bonache-Regidor C, Buzo-Franco D (2012) Can human disturbance promote nestedness? Songbirds and noise in urban parks as a case study. Landscape and Urban Planning, 104, 9-18.

Gutiérrez-Cánovas C, Millán A, Velasco J, Vaughan IP, Ormerod SJ (2013) Contrasting effects of natural and anthropogenic stressors on beta diversity in river organisms. Global Ecology and Biogeography, 22, 796-805.

Li CL, Zhao BB, Wang YP (2019) Nestedness of waterbird assemblages in the subsidence wetlands recently created by underground coal mining. Current Zoology, 65, 155-163.

Liu JJ, Coomes DA, Gibson L, Hu G, Liu JL, Luo YQ, Wu CP, Yu MJ (2019) Forest fragmentation in China and its effect on biodiversity. Biological Reviews, 94, 1636-1657.

MacKinnon J, Phillipps K, He FQ (2000) A Field Guide to the Birds of China. Hunan Education Publishing House, Changsha. (in Chinese) [约翰.马敬能, 卡伦·菲利普斯, 何 芬奇 (2000) 中国鸟类野外手册. 湖南教育出版社, 长 沙.]

Matthews TJ, Cottee-Jones HEW, Whittaker RJ (2015) Quantifying and interpreting nestedness in habitat islands: A synthetic analysis of multiple datasets. Diversity and Distributions, 21, 392-404.
Miller-Rushing AJ, Primack RB, Devictor V, Corlett RT, Cumming GS, Loyola R, Maas B, Pejchar L (2019) How does habitat fragmentation affect biodiversity? A controversial question at the core of conservation biology. Biological Conservation, 232, 271-273.

Patterson BD, Atmar W (1986) Nested subsets and the structure of insular mammalian faunas and archipelagos. Biological Journal of the Linnean Society, 28, 65-82.

Research and Design Institute of Environmental Science of Guizhou Province (2010) The Current Status of Biodiversity in Guizhou Province. Guizhou Science and Technology Press, Guiyang. (in Chinese) [贵州省环境科学研究设计院 (2010) 贵州省生物多样性现状. 贵州科技出版社, 贵阳.]

Si XF (2014) Species Turnover and Beta Diversity of Breeding Bird Communities in Fragmented Habitats. PhD dissertation, Zhejiang University, Hangzhou. (in Chinese with English abstract) [斯幸峰 (2014) 片段化生境中繁殖 鸟类群落的物种周转与 $\beta$ 多样性. 博士学位论文, 浙江大 学, 杭州.]

Wagner MA, Reynolds JD (2019) Salmon increase forest bird abundance and diversity. PLoS ONE, 14, e0210031.

Wang BY, Wang XM, Wang TH, Ding YZ (2012) Nested analysis of urban woodlot bird communities in Minhang District of Shanghai. Acta Ecologica Sinica, 32, 2788-2795. (in Chinese with English abstract) [王本耀, 王小明, 王天 厚, 丁由中 (2012) 上海闵行区园林鸟类群落嵌套结构. 生态学报, 32, 2788-2795.]

Wang YP, Bao YX, Yu MJ, Xu GF, Ding P (2010) Nestedness for different reasons: The distributions of birds, lizards and small mammals on islands of an inundated lake. Diversity and Distributions, 16, 862-873.

Wang YP, Ding P, Chen SH, Zheng GM (2013) Nestedness of bird assemblages on urban woodlots: Implications for conservation. Landscape and Urban Planning, 111, 59-67.

Watling JI, Donnelly MA (2006) Fragments as islands: A synthesis of faunal responses to habitat patchiness. Conservation Biology, 20, 1016-1025.

Wilson EO, MacArthur RH (1967) The Theory of Island Biogeography. Princeton University Press, Princeton.

Wu YR, Si XF, Chen CW, Zeng D, Zhao YH, Li JQ, Ding P (2016) Effects of dispersal abilities on community dynamics of breeding birds on the land-bridge islands in the Thousand Island Lake, China. Biodiversity Science, 24, 1135-1145. (in Chinese with English abstract) [吴奕如, 斯幸峰, 陈传 武, 曾頔, 赵郁豪, 李家琦, 丁平 (2016) 千岛湖陆桥岛 屿繁殖鸟类的扩散能力差异对群落动态的影响. 生物多 样性, 24, 1135-1145.]

Wu QQ, Liang ZS, Liu JJ, Yu MJ, Hu G (2017) Effects of habitat fragmentation on biodiversity in China. Chinese Journal of Ecology, 36, 2605-2614. (in Chinese with English abstract) [吴倩倩, 梁宗锁, 刘佳佳, 于明坚, 胡广 (2017) 中国生境片段化对生物多样性影响研究进展. 生 态学杂志, 36, 2605-2614.] 
Xu AC, Han XF, Zhang XM, Millien V, Wang YP (2017) Nestedness of butterfly assemblages in the Zhoushan Archipelago, China: Area effects, life-history traits and conservation implications. Biodiversity and Conservation, 26, 1375-1392.

Zhang JC, Wang YP, Jiang PP, Li P, Yu MJ, Ding P (2008) Nested analysis of Passeriform bird assemblages in the Thousand Island Lake region. Biodiversity Science, 16, 321-331. (in Chinese with English abstract) [张竞成, 王彦 平，蒋萍萍，李鹏，于明坚，丁平 (2008) 千岛湖雀形目 鸟类群落嵌套结构分析. 生物多样性, 16, 321-331.]

Zhang MH, Ma JZ (2014) A theoretical review of wildlife habitat fragmentation. Chinese Journal of Wildlife, 35, 6-14. (in Chinese with English abstract) [张明海, 马建章 (2014) 野生动物生境破碎化理论探讨. 野生动物学报, 35, 6-14.]

Zhang RZ (2011) Zoogeography of China. Science Press, Beijing. (in Chinese) [张荣祖 (2011) 中国动物地理. 科学 出版社, 北京.]

Zheng GM (2017) A Checklist on the Classification and Distribution of the Birds of China, 3rd edn. Science Press, Beijing. (in Chinese) [郑光美 (2017) 中国鸟类分类与分 布名录(第三版). 科学出版社, 北京.]

(责任编委: 王彦平 责任编辑: 问文杰)

\section{附录 Supplementary Material}

附录1 贵州花溪大学城26个林地斑块的地理特征、样线数量与长度和记录的鸟类物种数

Appendix 1 Characteristics of 26 fragmented woodlots, number and length of line-transects and number of bird species recorded in Huaxi University Town, Guizhou Province

https://www.biodiversity-science.net/fileup/PDF/2020336-1.pdf

附录2 贵州花溪大学城26个林地斑块中物种多度排列矩阵

Appendix 2 Species-by-sites abundance matrix of birds recorded on 26 fragmented woodlots in Huaxi University Town, Guizhou Province https://www.biodiversity-science.net/fileup/PDF/2020336-2.pdf 
郑进风, 唐蓉, 贺霜, 陈月红, 伍素, 张凯, 徐雨, 邹晓 (2021) 贵州花溪大学城破碎化林地鸟类多样性与嵌套分布格局. 生物多样性, 29, 661-667. http://www.biodiversity-science.net/CN/10.17520/biods.2020336

附录 1 贵州花溪大学城 26 个林地斑块的地理特征、样线数量与长度和记录的鸟类物种数

Appendix 1 Characteristics of 26 fragmented woodlots, number and length of line-transects and number of bird species recorded in Huaxi University Town, Guizhou Province

\begin{tabular}{|c|c|c|c|c|c|}
\hline $\begin{array}{l}\text { 林地斑块编号 } \\
\text { Woodlot no. }\end{array}$ & $\begin{array}{l}\text { 面积 } \\
\text { Area (ha) }\end{array}$ & $\begin{array}{l}\text { 距最近林地斑块距离 } \\
\text { Distance to the nearest } \\
\text { woodlot }(\mathrm{m})\end{array}$ & $\begin{array}{l}\text { 样线数量 } \\
\text { Number of line- } \\
\text { transects }\end{array}$ & $\begin{array}{l}\text { 样线长度 } \\
\text { Length of line- } \\
\text { transects (m) }\end{array}$ & $\begin{array}{l}\text { 记录的物种数* } \\
\text { Number of } \\
\text { species recorded }\end{array}$ \\
\hline 1 & 2.4 & 44.2 & 1 & 442.6 & 18 \\
\hline 2 & 6.2 & 20.0 & 1 & 915.5 & 22 \\
\hline 3 & 9.4 & 43.1 & 1 & 926.3 & 30 \\
\hline 4 & 3.6 & 56.5 & 1 & 336.3 & 15 \\
\hline 5 & 2.1 & 110.3 & 1 & 298.6 & 14 \\
\hline 6 & 0.9 & 23.4 & 1 & 172.1 & 16 \\
\hline 7 & 0.6 & 43.1 & 1 & 126.2 & 12 \\
\hline 8 & 8.6 & 20.0 & 1 & 617.1 & 22 \\
\hline 9 & 2.6 & 97.4 & 1 & 282.3 & 22 \\
\hline 10 & 3.7 & 33.3 & 1 & 392.8 & 16 \\
\hline 11 & 0.9 & 94.5 & 1 & 153.6 & 12 \\
\hline 12 & 2.3 & 273.3 & 1 & 293.2 & 12 \\
\hline 13 & 1.5 & 180.5 & 1 & 250.9 & 17 \\
\hline 14 & 1.5 & 10.8 & 1 & 254.1 & 23 \\
\hline 15 & 3.2 & 261.9 & 1 & 252.6 & 21 \\
\hline 16 & 1.2 & 91.2 & 1 & 159.1 & 16 \\
\hline 17 & 290.4 & 56.5 & 4 & $7,073.7$ & 47 \\
\hline 18 & 25.5 & 37.4 & 1 & $1,302.8$ & 28 \\
\hline 19 & 6.5 & 33.8 & 1 & 558.6 & 24 \\
\hline 20 & 19 & 33.8 & 1 & 892.2 & 33 \\
\hline 21 & 34.2 & 20.4 & 1 & $1,064.4$ & 26 \\
\hline 22 & 1.4 & 20.4 & 1 & 183.2 & 12 \\
\hline 23 & 1.1 & 85.1 & 1 & 204.5 & 15 \\
\hline 24 & 1.4 & 174.5 & 1 & 244.3 & 18 \\
\hline 25 & 11.5 & 10.8 & 1 & 585.4 & 37 \\
\hline 26 & 0.3 & 47.7 & 1 & 82.5 & 16 \\
\hline
\end{tabular}

*未包括燕子、雨燕、水鸟等高空飞行或非森林鸟类以及调查中仅记录到1次的偶然出现物种。

* Fly-overs (e.g., swallows and swifts), non-forest dwelling birds (e.g., waterbirds), and birds that were only recorded once in the survey were excluded. 
附录 2 贵州花溪大学城 26 个林地斑块中物种多度排列矩阵

Appendix 2 Species-by-sites abundance matrix of birds recorded on 26 fragmented woodlots in Huaxi University Town, Guizhou Province

\begin{tabular}{|c|c|c|c|c|c|c|c|c|c|c|c|c|c|c|c|c|c|c|c|c|c|c|c|c|c|c|}
\hline \multirow[t]{2}{*}{$\begin{array}{c}\text { 物种 } 2 \\
\text { Species }\end{array}$} & \multicolumn{26}{|c|}{$\begin{array}{c}\text { 林地斑块编号 } \\
\text { Woodlot no. }\end{array}$} \\
\hline & 1 & 2 & 3 & 4 & 5 & 6 & 7 & 8 & 9 & 10 & 11 & 12 & 13 & 14 & 15 & 16 & 17 & 18 & 19 & 20 & 21 & 22 & 23 & 24 & 25 & 26 \\
\hline 黄慰䡚 Pycnonotus xanthorrhous & 69 & 57 & 62 & 68 & 64 & 45 & 34 & 62 & 41 & 32 & 41 & 21 & 57 & 33 & 67 & 53 & 294 & 87 & 25 & 73 & 102 & 29 & 30 & 46 & 40 & 9 \\
\hline 白㚘噪鵰 Garrulax sannio & 67 & 48 & 69 & 27 & 26 & 19 & 29 & 27 & 38 & 22 & 24 & 18 & 54 & 9 & 28 & 24 & 109 & 38 & 15 & 37 & 51 & 9 & 12 & 6 & 18 & 5 \\
\hline 北红尾鸲 Phoenicurus auroreus & 5 & 14 & 25 & 10 & 6 & 18 & 5 & 8 & 9 & 8 & 7 & 6 & 12 & 8 & 15 & 22 & 88 & 30 & 5 & 15 & 22 & 15 & 21 & 5 & 17 & 5 \\
\hline 麻雀 Passer montanus & 15 & 7 & 20 & 17 & 7 & 16 & 3 & 40 & 8 & 3 & 15 & 32 & 21 & 21 & 46 & 4 & 95 & 53 & 89 & 59 & 69 & 58 & 59 & 23 & 70 & 10 \\
\hline 白䴖鸰 Motacilla alba & 7 & 9 & 9 & 2 & 7 & 2 & 10 & 19 & 3 & 7 & 3 & 4 & 4 & 4 & 6 & 19 & 29 & 10 & 4 & 12 & 15 & 9 & 6 & 6 & 13 & 5 \\
\hline 领雀嘴軼 Spizixos semitorques & 16 & 21 & 8 & 14 & 6 & 2 & 5 & 30 & 12 & 12 & 9 & 17 & 19 & 7 & 18 & 15 & 73 & 37 & 13 & 21 & 54 & 0 & 9 & 7 & 37 & 5 \\
\hline 山斑鸠 Streptopelia orientalis & 5 & 11 & 4 & 1 & 1 & 7 & 5 & 2 & 10 & 1 & 3 & 1 & 2 & 6 & 6 & 2 & 10 & 4 & 3 & 4 & 5 & 0 & 0 & 1 & 12 & 2 \\
\hline 红嘴蓝鹊 Urocissa erythrorhyncha & 3 & 8 & 5 & 7 & 2 & 3 & 3 & 8 & 7 & 6 & 1 & 15 & 7 & 4 & 0 & 17 & 18 & 4 & 5 & 2 & 16 & 0 & 2 & 0 & 9 & 16 \\
\hline 大山雀 Parus cinereus & 13 & 26 & 7 & 3 & 1 & 1 & 7 & 17 & 7 & 18 & 0 & 0 & 3 & 8 & 3 & 5 & 19 & 8 & 5 & 5 & 6 & 1 & 2 & 0 & 12 & 3 \\
\hline 金翅雀 Chloris sinica & 1 & 24 & 17 & 10 & 9 & 12 & 0 & 2 & 13 & 12 & 3 & 3 & 4 & 6 & 6 & 0 & 24 & 17 & 0 & 5 & 23 & 2 & 18 & 3 & 15 & 1 \\
\hline 喜鹊 Pica pica & 0 & 10 & 3 & 3 & 1 & 8 & 0 & 6 & 6 & 2 & 0 & 0 & 7 & 3 & 3 & 0 & 19 & 15 & 3 & 2 & 4 & 4 & 2 & 9 & 3 & 4 \\
\hline 棕背伯劳 Lanius schach & 0 & 1 & 2 & 1 & 0 & 2 & 3 & 1 & 1 & 1 & 0 & 7 & 2 & 0 & 3 & 1 & 20 & 7 & 0 & 2 & 2 & 1 & 0 & 1 & 2 & 0 \\
\hline 强脚树莺 Horornis fortipes & 0 & 0 & 9 & 0 & 5 & 0 & 0 & 1 & 1 & 1 & 0 & 2 & 1 & 0 & 3 & 4 & 52 & 2 & 0 & 9 & 21 & 0 & 7 & 2 & 5 & 0 \\
\hline 鹊鸩 Copsychus saularis & 1 & 1 & 2 & 0 & 0 & 0 & 1 & 1 & 4 & 0 & 0 & 0 & 2 & 10 & 2 & 0 & 8 & 4 & 6 & 1 & 1 & 0 & 0 & 0 & 5 & 0 \\
\hline 暗绿绣眼鸟 Zosterops japonicus & 5 & 14 & 10 & 11 & 2 & 2 & 0 & 0 & 7 & 0 & 0 & 0 & 0 & 0 & 0 & 0 & 47 & 8 & 0 & 11 & 5 & 0 & 0 & 0 & 9 & 0 \\
\hline 红头长尾山雀 Aegithalos concinnus & 2 & 22 & 16 & 0 & 0 & 1 & 0 & 0 & 6 & 6 & 0 & 0 & 0 & 1 & 0 & 0 & 31 & 20 & 0 & 0 & 3 & 0 & 0 & 0 & 1 & 0 \\
\hline 八哥 Acridotheres cristatellus & 0 & 0 & 1 & 0 & 0 & 0 & 0 & 2 & 0 & 0 & 0 & 2 & 0 & 0 & 1 & 0 & 15 & 4 & 0 & 1 & 5 & 0 & 0 & 0 & 7 & 0 \\
\hline 黄腰柳莺 Phylloscopus proregulus & 9 & 15 & 6 & 0 & 2 & 4 & 5 & 0 & 0 & 0 & 0 & 0 & 0 & 0 & 0 & 0 & 2 & 0 & 0 & 4 & 0 & 0 & 0 & 0 & 3 & 0 \\
\hline 棕脸叙鸟莺 Abroscopus albogularis & 1 & 5 & 0 & 0 & 0 & 0 & 0 & 1 & 0 & 1 & 0 & 0 & 0 & 0 & 0 & 1 & 4 & 1 & 0 & 0 & 0 & 0 & 0 & 0 & 3 & 0 \\
\hline 纯色山鹪莺 Prinia inornata & 0 & 0 & 0 & 0 & 0 & 0 & 0 & 0 & 0 & 0 & 1 & 0 & 0 & 0 & 8 & 0 & 9 & 1 & 0 & 4 & 1 & 4 & 1 & 10 & 0 & 0 \\
\hline 虎纹伯劳 Lanius tigrinus & 0 & 0 & 0 & 0 & 0 & 1 & 0 & 6 & 1 & 0 & 0 & 0 & 0 & 1 & 0 & 0 & 1 & 0 & 6 & 0 & 0 & 0 & 0 & 1 & 10 & 0 \\
\hline 乌冻 Turdus mandarinus & 0 & 0 & 2 & 0 & 0 & 0 & 0 & 0 & 0 & 0 & 0 & 0 & 0 & 3 & 0 & 0 & 3 & 1 & 3 & 3 & 0 & 0 & 0 & 0 & 18 & 1 \\
\hline 珠颈斑鸭 Spilopelia chinensis & 0 & 5 & 1 & 0 & 0 & 0 & 0 & 0 & 0 & 0 & 0 & 0 & 1 & 0 & 0 & 0 & 2 & 1 & 8 & 5 & 0 & 0 & 0 & 0 & 0 & 0 \\
\hline 红尾伯劳 Lanius cristatus & 1 & 0 & 0 & 0 & 0 & 0 & 0 & 3 & 0 & 0 & 0 & 0 & 2 & 3 & 0 & 0 & 1 & 0 & 0 & 0 & 0 & 0 & 0 & 1 & 1 & 0 \\
\hline 黑喉石鵖 Saxicola torquata & 0 & 0 & 0 & 0 & 0 & 0 & 0 & 0 & 0 & 0 & 0 & 0 & 0 & 0 & 20 & 0 & 21 & 0 & 0 & 2 & 8 & 7 & 10 & 16 & 0 & 0 \\
\hline
\end{tabular}




\begin{tabular}{|c|c|c|c|c|c|c|c|c|c|c|c|c|c|c|c|c|c|c|c|c|c|c|c|c|c|c|}
\hline \multirow[t]{2}{*}{$\begin{array}{l}\text { 物种 }^{2} \\
\text { Species }\end{array}$} & \multicolumn{26}{|c|}{$\begin{array}{l}\text { 林地斑块编号 } \\
\text { Woodlot no. }\end{array}$} \\
\hline & 1 & 2 & 3 & 4 & 5 & 6 & 7 & 8 & 9 & 10 & 11 & 12 & 13 & 14 & 15 & 16 & 17 & 18 & 19 & 20 & 21 & 22 & 23 & 24 & 25 & 26 \\
\hline 白腰文鸟 Lonchura striata & 0 & 0 & 0 & 0 & 0 & 0 & 0 & 0 & 2 & 0 & 0 & 0 & 0 & 0 & 0 & 0 & 1 & 0 & 1 & 1 & 2 & 2 & 0 & 1 & 0 & $\overline{0}$ \\
\hline 黑尾蜡嘴雀 Eophona migratoria & 0 & 0 & 1 & 0 & 0 & 0 & 0 & 4 & 7 & 0 & 0 & 0 & 0 & 0 & 0 & 2 & 2 & 0 & 0 & 0 & 0 & 0 & 0 & 0 & 13 & 3 \\
\hline 噪鹃 Eudynamys scolopacea & 0 & 0 & 4 & 0 & 0 & 0 & 0 & 0 & 0 & 0 & 0 & 0 & 0 & 2 & 0 & 0 & 0 & 1 & 0 & 1 & 0 & 0 & 0 & 0 & 5 & 1 \\
\hline 粉红山椒鸟 Pericrocotus roseus & 0 & 3 & 0 & 0 & 0 & 0 & 0 & 4 & 0 & 0 & 4 & 0 & 0 & 0 & 0 & 0 & 1 & 0 & 1 & 0 & 0 & 0 & 0 & 0 & 3 & 0 \\
\hline 黑胸斩 Turdus dissimilis & 0 & 0 & 0 & 0 & 0 & 0 & 0 & 0 & 7 & 0 & 0 & 0 & 0 & 2 & 0 & 0 & 1 & 0 & 1 & 0 & 0 & 0 & 0 & 0 & 20 & 2 \\
\hline 蓝矶鸫 Monticola solitarius & 4 & 0 & 2 & 0 & 0 & 0 & 0 & 2 & 0 & 0 & 0 & 0 & 0 & 0 & 0 & 0 & 0 & 1 & 2 & 0 & 0 & 0 & 4 & 0 & 0 & 0 \\
\hline 紫啸冻 Myophonus caeruleus & 0 & 0 & 2 & 0 & 0 & 0 & 0 & 0 & 0 & 0 & 0 & 0 & 0 & 0 & 0 & 0 & 10 & 1 & 0 & 2 & 4 & 0 & 0 & 0 & 3 & 0 \\
\hline 褐胁雀䧓 Schoeniparus dubius & 0 & 6 & 6 & 3 & 0 & 0 & 0 & 0 & 1 & 0 & 0 & 0 & 0 & 0 & 0 & 0 & 3 & 0 & 0 & 0 & 0 & 0 & 0 & 0 & 2 & 0 \\
\hline 山麻雀 Passer cinnamomeus & 0 & 0 & 0 & 0 & 0 & 0 & 0 & 0 & 10 & 0 & 0 & 0 & 0 & 4 & 0 & 5 & 5 & 0 & 1 & 0 & 0 & 0 & 0 & 0 & 8 & 0 \\
\hline 灰䴖鸰 Motacilla cinerea & 0 & 0 & 0 & 0 & 0 & 0 & 0 & 0 & 0 & 0 & 0 & 0 & 0 & 0 & 0 & 1 & 1 & 0 & 1 & 1 & 2 & 0 & 0 & 0 & 0 & 0 \\
\hline 斑胸钩嘴扸 Erythrogenys gravivox & 0 & 0 & 0 & 0 & 0 & 0 & 0 & 0 & 0 & 0 & 0 & 0 & 0 & 2 & 0 & 0 & 12 & 0 & 0 & 1 & 5 & 0 & 0 & 3 & 0 & 0 \\
\hline 灰喉鸦雀 Paradoxornis alphonsiana & 0 & 0 & 1 & 0 & 0 & 0 & 0 & 0 & 0 & 0 & 0 & 0 & 0 & 1 & 0 & 0 & 11 & 0 & 1 & 0 & 1 & 0 & 0 & 0 & 1 & 0 \\
\hline 淡黄腰柳莺 Phylloscopus chloronotus & 0 & 0 & 2 & 0 & 0 & 0 & 0 & 1 & 0 & 0 & 0 & 0 & 0 & 0 & 3 & 0 & 5 & 0 & 2 & 0 & 0 & 0 & 0 & 0 & 0 & 0 \\
\hline 山鴡莺 Prinia crinigera & 0 & 0 & 0 & 0 & 0 & 0 & 0 & 0 & 0 & 0 & 1 & 0 & 0 & 0 & 1 & 0 & 8 & 2 & 0 & 1 & 1 & 0 & 1 & 0 & 0 & 0 \\
\hline 树翏鸟 Anthus hodgsoni & 0 & 0 & 0 & 0 & 0 & 0 & 0 & 0 & 0 & 0 & 0 & 0 & 0 & 3 & 0 & 1 & 2 & 0 & 0 & 0 & 0 & 0 & 0 & 1 & 0 & 0 \\
\hline 红喉姬䳜 Ficedula albicilla & 0 & 1 & 0 & 1 & 0 & 0 & 0 & 0 & 0 & 0 & 0 & 0 & 0 & 0 & 0 & 0 & 1 & 0 & 0 & 1 & 0 & 0 & 0 & 0 & 0 & 0 \\
\hline 斑文鸟 Lonchura punctulata & 0 & 0 & 0 & 0 & 0 & 0 & 0 & 0 & 0 & 0 & 0 & 0 & 0 & 0 & 1 & 0 & 0 & 2 & 0 & 11 & 13 & 0 & 0 & 0 & 0 & 0 \\
\hline 红头穗倜 Cyanoderma ruficeps & 0 & 0 & 1 & 0 & 0 & 0 & 0 & 0 & 0 & 0 & 0 & 0 & 0 & 0 & 0 & 0 & 4 & 0 & 0 & 3 & 0 & 0 & 0 & 0 & 0 & 1 \\
\hline 丝光椋鸟 Spodiopsar sericeus & 0 & 0 & 0 & 0 & 0 & 0 & 0 & 0 & 0 & 0 & 0 & 0 & 0 & 0 & 0 & 0 & 5 & 0 & 0 & 1 & 0 & 0 & 0 & 0 & 5 & 0 \\
\hline 寿带 Terpsiphone paradisi & 1 & 0 & 0 & 0 & 0 & 0 & 0 & 0 & 0 & 0 & 0 & 0 & 0 & 0 & 0 & 0 & 0 & 0 & 1 & 0 & 0 & 0 & 0 & 0 & 0 & 0 \\
\hline 棕颈钩嘴鹤 Pomatorhinus ruficollis & 0 & 0 & 1 & 0 & 0 & 0 & 0 & 0 & 0 & 0 & 0 & 0 & 0 & 0 & 0 & 0 & 9 & 1 & 0 & 0 & 0 & 0 & 0 & 0 & 0 & 0 \\
\hline 黄喉鸥 Emberiza elegans & 0 & 0 & 0 & 0 & 0 & 0 & 0 & 0 & 0 & 0 & 0 & 0 & 0 & 0 & 0 & 0 & 5 & 1 & 0 & 4 & 0 & 0 & 0 & 0 & 0 & 0 \\
\hline 发冠卷尾 Dicrurus hottentottus & 0 & 3 & 0 & 0 & 0 & 0 & 0 & 0 & 0 & 0 & 0 & 0 & 0 & 0 & 0 & 0 & 0 & 0 & 0 & 0 & 0 & 0 & 0 & 0 & 1 & 0 \\
\hline 松鸦 Garrulus glandarius & 0 & 0 & 0 & 0 & 0 & 0 & 0 & 0 & 0 & 0 & 0 & 0 & 0 & 3 & 0 & 0 & 1 & 0 & 0 & 0 & 0 & 0 & 0 & 0 & 0 & 0 \\
\hline 铜蓝叙 Eumyias thalassinus & 0 & 0 & 0 & 0 & 0 & 0 & 0 & 0 & 0 & 0 & 0 & 0 & 0 & 0 & 0 & 0 & 0 & 0 & 1 & 0 & 0 & 0 & 0 & 0 & 3 & 0 \\
\hline 北灰覀 Muscicapa dauurica & 0 & 0 & 0 & 0 & 0 & 0 & 0 & 0 & 0 & 0 & 0 & 0 & 1 & 0 & 0 & 0 & 0 & 0 & 0 & 0 & 0 & 0 & 0 & 0 & 1 & 0 \\
\hline 栗耳鸤 Yuhina castaniceps & 0 & 0 & 0 & 0 & 0 & 0 & 0 & 0 & 0 & 5 & 0 & 0 & 0 & 0 & 0 & 0 & 15 & 0 & 0 & 0 & 0 & 0 & 0 & 0 & 0 & 0 \\
\hline 灰眉岩鸥 Emberiza godlewskii & 0 & 0 & 1 & 0 & 0 & 0 & 0 & 0 & 0 & 0 & 0 & 0 & 0 & 0 & 0 & 0 & 1 & 0 & 0 & 0 & 0 & 0 & 0 & 0 & 0 & 0 \\
\hline
\end{tabular}




\begin{tabular}{|c|c|c|c|c|c|c|c|c|c|c|c|c|c|c|c|c|c|c|c|c|c|c|c|c|c|c|}
\hline \multirow[t]{2}{*}{$\begin{array}{l}\text { 物种 }^{2} \\
\text { Species }\end{array}$} & \multicolumn{26}{|c|}{$\begin{array}{c}\text { 林地斑块编号 } \\
\text { Woodlot no. } \\
\end{array}$} \\
\hline & 1 & 2 & 3 & 4 & 5 & 6 & 7 & 8 & 9 & 10 & 11 & 12 & 13 & 14 & 15 & 16 & 17 & 18 & 19 & 20 & 21 & 22 & 23 & 24 & 25 & 26 \\
\hline 环颈雉 Phasianus colchicus & 0 & 0 & 0 & 0 & 0 & 0 & 0 & 0 & 0 & 0 & 0 & 0 & 0 & 0 & 2 & 0 & 0 & 0 & 0 & 1 & 0 & 0 & 0 & 0 & 0 & 0 \\
\hline 栗耳鸥 Emberiza fucata & 0 & 0 & 0 & 0 & 0 & 0 & 0 & 0 & 0 & 0 & 0 & 0 & 0 & 0 & 3 & 0 & 1 & 0 & 0 & 0 & 0 & 0 & 0 & 0 & 0 & 0 \\
\hline 大拟啄木鸟 Psilopogon virens & 0 & 0 & 0 & 0 & 0 & 0 & 0 & 0 & 0 & 0 & 0 & 0 & 0 & 0 & 0 & 0 & 0 & 0 & 0 & 0 & 0 & 0 & 0 & 0 & 4 & 0 \\
\hline 灰头绿豚木鸟 Picus canus & 0 & 0 & 0 & 0 & 0 & 0 & 0 & 0 & 0 & 0 & 0 & 0 & 0 & 0 & 0 & 0 & 0 & 0 & 0 & 0 & 0 & 0 & 0 & 0 & 2 & 0 \\
\hline 蓝翅希鹋 Siva cyanouroptera & 0 & 0 & 0 & 0 & 0 & 0 & 0 & 0 & 0 & 0 & 0 & 0 & 0 & 0 & 0 & 0 & 0 & 0 & 0 & 0 & 0 & 0 & 0 & 0 & 3 & 0 \\
\hline
\end{tabular}

1表中的物种多度是10次调查中记录的个体数的累积值 The abundance is the accumulative records of individuals of each species during ten surveys.

2未包括燕子、雨燕、水鸟等高空飞行或非森林鸟类以及调查中仅记录到1次的偶然出现物种 Fly-overs (e.g., swallows and swifts), non-forest dwelling birds (e.g., waterbirds), and birds that were only recorded once in the survey were excluded. 